\title{
Symmetries and nonequilibrium thermodynamics
}

\author{
Sergio Bordel* \\ Laboratory of Cell Culture, Institute of Cardiology, Lithuanian University of Health Sciences, Sukileliu ave. 17, Kaunas, Lithuania \\ and Department of Chemical and Biological Engineering, Chalmers University of Technology, Kemivägen 10, SE-41296 Göteborg, Sweden
}

(Received 6 March 2017; revised manuscript received 27 April 2017; published 8 June 2017)

\begin{abstract}
Thermodynamic systems can be defined as composed by many identical interacting subsystems. Here it is shown how the dynamics of relaxation toward equilibrium of a thermodynamic system is closely related to the symmetry group of the Hamiltonian of the subsystems of which it is composed. The transitions between states driven by the interactions between identical subsystems correspond to elements of the root system associated to the symmetry group of their Hamiltonian. This imposes constraints on the relaxation dynamics of the complete thermodynamic system, which allow formulating its evolution toward equilibrium as a system of linear differential equations in which the variables are the thermodynamic forces of the system. The trajectory of a thermodynamic system in the space of thermodynamic forces corresponds to the negative gradient of a potential function, which depends on the symmetry group of the Hamiltonian of the individual interacting subsystems.
\end{abstract}

DOI: 10.1103/PhysRevE.95.062108

\section{INTRODUCTION}

Nonequilibrium thermodynamics is still open to controversy and different interpretations [1]. General principles describing the relaxation of a system toward thermodynamic equilibrium or the steady states of a system that is kept away from equilibrium due to its constant exchange of matter or energy with its environment, have been searched with variable success. Prigogine [2] and Ziegler [3] proposed, respectively, minimal and maximal entropy production rates characterizing steady states, the first one related to global entropy production under certain boundary conditions and the second one being local [4]. Other authors [5] have proposed generalizations far away from the equilibrium to Onsager's relations between thermodynamic fluxes and forces. In previous works, we argued that Onsager's reciprocity relations are equivalent to a principle of steepest entropy increase [6,7]. The same conclusions had been previously reached by Beretta [8-10] by introducing a dissipative term in Schrödinger's equation. Other authors have also explained the irreversible relaxation of thermodynamic systems toward equilibrium by modifying Schrödinger's equation [11-14]. In fact, the apparent contradiction between the second principle of thermodynamics and the time reversibility of Schrödinger's equation can be explained based on the theory of quantum measurement (the projection of a wave function on an eigenvector of the operator associated to a measured observable) and von Neumann's entropy definition. Indeed, while von Neumann's entropy does not change over time when the system evolves following Schrödinger's equation, it can change if the system is projected on one eigenvector of some observable with an associated operator that does not commute with the Hamiltonian. A recent explanation following these lines can be found in Ref. [15]. Fermi's golden rule [16] describing transitions between states (different eigenvalues of a Hamiltonian) as a result of a perturbation, uses implicitly the theory of quantum measurement to explain the observed transitions.

\footnotetext{
*sergio_bordel@hotmail.com
}

In this work, we present the derivation of a general master equation describing the relaxation of an isolated system toward equilibrium. A thermodynamic system is defined as being composed of identical subsystems that interact weakly between each other (which allows using Fermi's golden rule [16] to describe the transitions between states of each subsystem). The only initial assumptions used in the derivation are Fermi's golden rule and the definition of the entropy production rate as the product of thermodynamic fluxes and forces [7], which allows us to define a nonequilibrium statistical distribution (Appendix E) without any of the extra assumptions frequently used, such as the maximization of the information entropy $[17,18]$. Thermodynamic fluxes are defined as the variation rates of certain observables, which are associated to quantum operators that commute with the Hamiltonian of the individual subsystems.

The results in this paper are very wide but not completely general, because we are treating the case of isolated systems formed by identical subsystems with the same symmetries. This would not cover, for instance, the approach to equilibrium between isotropic fluids and crystalline solids or liquid crystals etc., which involve the interactions between systems with different symmetries. However, the present formalism is expected to be generalized also to these more general situations (in particular, the relaxation of systems in contact with an isothermal bath), and to clarify the influence of the different symmetries on the coefficients describing the respective energy exchange among such different degrees of freedom, in the light of Appendix A to the paper. However, this would require a full paper and will be done in the future.

In this article, we show how the symmetries of the Hamiltonian of the individual subsystems impose stringent rules on the allowed transitions between states and their relative rates. The states in which each subsystem can be found are defined by its energy level (each energy level corresponds to a representation of the symmetry group of the Hamiltonian) and a vector of weights, which are eigenvalues of a subset of generators of the symmetry group (the Cartan subalgebra). It is proven that the only allowed transitions between states (in a system formed by indistinguishable subsystems) correspond to elements of the root system associated to the Hamiltonian's 
symmetry group. All these properties allow describing the trajectory of the system in the space of thermodynamic forces, by a system of linear differential equations whose coefficients can be obtained from the Hamiltonian's symmetry group and the interaction potential between pairs of subsystems.

\section{FORMALISM}

\section{A. Transitions between states in a system of many identical weakly interacting subsystems}

We consider a system formed by a number $s$ of identical subsystems (for example, a container filled with $s$ molecules of a certain chemical compound). To be as general as possible, the subsystems could be large groups of particles or molecules that form a Gibbs ensemble. The only necessary criteria for these subsystems is that they are identical to each other. Each subsystem $i$ is characterized by a Hamiltonian operator $h_{i}$; as all the subsystems are identical, their different Hamiltonians have the same form and they only differ on the variables that they act on (the position and momentum coordinates of the components of each subsystem). We will use the following notation for the eigenvectors of the Hamiltonian $h_{i}:\left|i_{k}\right\rangle$, so that

$$
h_{i}\left|i_{k}\right\rangle=\varepsilon_{k}\left|i_{k}\right\rangle \text {. }
$$

In Eq. (1), $\varepsilon_{k}$ is a real eigenvalue of $h_{i}$. As the Hamiltonians of the different subsystems have the same form, the values $\varepsilon_{k}$ are the same for each subsystem and therefore do not depend on $i$. The different eigenvalues $\varepsilon_{k}$ correspond to the different energy levels in which a subsystem can be observed and the set of eigenvectors $\left\{\left|i_{k}\right\rangle\right\}$ forms an orthonormal basis of the Hilbert space $H_{i}$ that contains all the possible wave functions $\psi(i)$ describing the state of the subsystem $i$ :

$$
\psi(i)=\sum_{k} c_{i k}\left|i_{k}\right\rangle .
$$

The wave functions describing the whole system are elements of the Hilbert space $H_{S}$, resulting from the tensor product of the Hilbert spaces $H_{i}$ :

$$
H_{S}=\prod_{i=1}^{s} H_{i} .
$$

An orthonormal basis of the space $H_{S}$ is formed by the tensor products of the elements of the bases of $H_{i}$. We will denote the elements of this basis as

$$
|\sigma\rangle=\left|1_{k_{1}}\right\rangle\left|2_{k_{2}}\right\rangle \ldots\left|s_{k_{s}}\right\rangle .
$$

The symbol $\sigma$ designates an $s$-tuple $k_{1} k_{2} \ldots k_{s}$ of indexes that correspond to eigenvectors of $h_{i}$. Now we can define $N_{k}$ as the number of times the index $k$ is repeated in the $s$-tuple $\sigma$, or in other words, the number of subsystems in the state $k$.

Our thermodynamic system has been defined as formed by identical subsystems, which makes them fundamentally indistinguishable from each other. In this case, the wave function describing the whole system has to be symmetric with respect to the permutation of two subsystems. Even if the system is composed of fermions, we can define the subsystems as pairs of fermions; therefore, we can assume symmetric wave functions without loss of generality. Symmetric normalized wave functions will take the following form:

$$
|S\rangle=\sqrt{\frac{\prod_{k} N_{k} !}{s !}} \sum_{p(\sigma)}|p(\sigma)\rangle,
$$

where $p(\sigma)$ is a permutation of the indexes in the $s$-tuple $\sigma$.

The Hamiltonian of the whole system, including the interactions between identical subsystems, will take the form

$$
\hat{H}=\sum_{i} h_{i}+\sum_{i, j} \frac{1}{2} \hat{V}_{i j}=\hat{H}_{0}+\hat{V} .
$$

For weak interactions between the subsystems, the transition frequency between two states can be described by Fermi's golden rule:

$$
W_{S_{0} \rightarrow S_{f}}=\frac{2 \pi}{\hbar}\left\langle S_{f}|\hat{V}| S_{0}\right\rangle^{2} \delta\left(E_{S_{f}}-E_{S_{0}}\right) .
$$

As shown in Appendix A [Eq. (A5)], the frequency of transitions involving changes in the state of more than two subsystems simultaneously is equal to zero. As the subsystems are undistinguishable, there is no difference between a situation in which a subsystem in the state $r$ transits to state $r^{\prime}$ and a subsystem in state $l$ transits to state $l^{\prime}$ or a subsystem in state $r$ transits to state $l^{\prime}$ and a subsystem in state $l$ transits to state $r^{\prime}$. Therefore, we can just talk about $l r \rightarrow l^{\prime} r^{\prime}$ transitions, whose frequency is equal to

$$
T_{r l \rightarrow r^{\prime} l^{\prime}}=\left(\frac{\prod_{k} N_{k}}{s !}\right)^{2} \Gamma_{r l \rightarrow r^{\prime} l^{\prime}} N_{r} N_{l}\left(N_{r^{\prime}}+1\right)\left(N_{l^{\prime}}+1\right),
$$

where

$$
\Gamma_{r l \rightarrow r^{\prime} l^{\prime}}=\Gamma_{r^{\prime} l^{\prime} \rightarrow r l}=\frac{2 \pi}{\hbar}\left\langle l^{\prime} ; r^{\prime}\left|\hat{V}_{i j}\right| r ; l\right\rangle^{2} \delta\left(\varepsilon_{r^{\prime}}+\varepsilon_{l^{\prime}}-\varepsilon_{r}-\varepsilon_{l}\right),
$$

$$
|r ; l\rangle=\frac{1}{\sqrt{2}}\left(\left|i_{r}\right\rangle\left|j_{l}\right\rangle+\left|i_{l}\right\rangle\left|j_{r}\right\rangle\right) .
$$

From now on the indexes identifying the subsystems $(i, j)$ will not be used. The energy of each state is referred as $\varepsilon$ in Eq. (9).

Equation (8) is derived in Appendix B, and in Appendix D it is also shown that it leads to a Bose-Einstein equilibrium distribution.

\section{B. Symmetries and states of a quantum system}

In this section, we remind some general facts about the description of a quantum system in terms of its symmetries, which are textbook material [19]. So far, the eigenstates of the Hamiltonian $h_{i}$ have been described using the indexes $r$ and $l$. In fact, these eigenstates can be described in terms of the symmetry group of the Hamiltonian. The symmetry group of $h_{i}$ is a Lie group whose generators form a Lie algebra and commute with the Hamiltonian $h_{i}$. Among the generators of the symmetry group it is possible to choose a maximal subset of generators that commute with each other (the Cartan sub-algebra). The elements of the Cartan subalgebra will be noted as $\hat{A}_{i}$ :

$$
\left[\hat{A}_{i}, \hat{A}_{j}\right]=0 .
$$


The operators in the Cartan algebra have the same eigenvectors, as they commute with each other. The rest of the generators of the Lie algebra, which do not commute with the elements of the Cartan algebra, can be chosen to be eigenvectors of the elements of the Cartan algebra under the operation of commutation. They satisfy the following commutation relations:

$$
\left[\hat{A}_{i}, \hat{E}_{\vec{\alpha}}\right]=\alpha_{i} \hat{E}_{\vec{\alpha}}
$$

The vector $\vec{\alpha}$ contains the eigenvalues of each element of the Cartan algebra and it is referred to as a root vector. The root vectors are defined by the symmetry of the system.

Each energy level of the Hamiltonian $h_{i}$ is a subspace of the Hilbert space $H_{i}$ with as many dimensions as the degeneracy of the energy level. It is possible to choose an orthonormal basis of this subspace, in which each of its members is an eigenvector of the operators in the Cartan subalgebra. The corresponding eigenvalues form a so-called weight vector $\vec{\mu}$, which can be used to indicate each state within an energy level. The subspace corresponding to an energy level is a G-module of a representation of the symmetry group of the Hamiltonian. Each energy level corresponds to a group representation and can be denoted by the vector $\vec{M}$, which is equal to the highest weight vector within the energy level. Therefore, each state of the system can be identified by the pair of vectors $\vec{\mu}$ and $\vec{M}$. The operators that form the Lie algebra transform the states of the system as follows:

$$
\begin{gathered}
\hat{A}_{i}|\vec{\mu} \vec{M}\rangle=\mu_{i}|\vec{\mu} \vec{M}\rangle, \\
\hat{E}_{\vec{\alpha}}|\vec{\mu} \vec{M}\rangle=U_{\vec{\alpha}, \vec{\mu}}|(\vec{\mu}+\vec{\alpha}) \vec{M}\rangle .
\end{gathered}
$$

The operator $\hat{E}_{\vec{\alpha}}$ is a ladder operator that displaces the vector of weights $\vec{\mu}$ by adding to it the root vector $\vec{\alpha}$. Starting from $\vec{\mu}$ it is possible to operate with $\hat{E}_{\vec{\alpha}} p$ times until one of the weights $\mu_{i}$ reaches its maximal value $M_{i}$ (or minimal value $-M_{i}$ ) for the considered energy level. Once this limit has been reached, the operator $\hat{E}_{\vec{\alpha}}$ will transform the final state in a zero value:

$$
\hat{E}_{\vec{\alpha}}|(\vec{\mu}+p \vec{\alpha}) \vec{M}\rangle=0 .
$$

The coefficients $U_{\vec{\alpha}, \vec{\mu}}$ depend on $\vec{\mu}, \vec{\alpha}$, and $p$ in the following way:

$$
U_{\vec{\alpha}, \vec{\mu}}=\sqrt{\frac{1}{2} \vec{\alpha}^{2} p\left(\frac{2 \vec{\mu} \cdot \vec{\alpha}}{\vec{\alpha} \cdot \vec{\alpha}}+p+1\right)} .
$$

The maximal number of times in which it is possible to apply the operator $\hat{E}_{-\vec{\alpha}}$ on the state $\vec{\mu}$ is equal to

$$
q=\frac{2 \vec{\mu} \cdot \vec{\alpha}}{\vec{\alpha} \cdot \vec{\alpha}}+p
$$

We point out two useful relations (derived from the previous two equations) to be used later:

$$
\begin{gathered}
\left(U_{\vec{\alpha},(\vec{\mu}-\vec{\alpha})}\right)^{2}-\left(U_{\vec{\alpha}, \vec{\mu}}\right)^{2}=\vec{\mu} \cdot \vec{\alpha}, \\
U_{\vec{\alpha}, \vec{\mu}}=U_{-\vec{\alpha},(\vec{\mu}+\vec{\alpha}) .}
\end{gathered}
$$

As the coefficients depend on the integer $p$, which itself depends on the representation, strictly speaking we should also introduce the index $\vec{M}$ in the notation $U_{\vec{\alpha}, \vec{\mu} \vec{M}}$.

\section{Symmetry properties of two identical quantum systems}

To describe the relaxation of a thermodynamic system, we are interested in computing the expressions $\left\langle l^{\prime} ; r^{\prime}\left|\hat{V}_{i j}\right| r ; l\right\rangle$ that appear in Eq. (9). To achieve this goal, it is necessary to find the generators of the symmetry group of a combination of two indistinguishable systems. As both systems are fundamentally indistinguishable, the symmetry operations should act identically on both; therefore, the symmetry group of the combined system is the same as the symmetry group of a single system. The generators of the symmetry group are just the sums of the generators acting on each individual system:

$$
\begin{aligned}
& \hat{A}_{i}=\hat{A}_{i}^{1}+\hat{A}_{i}^{2}, \\
& \hat{E}_{\vec{\alpha}}=\hat{E}_{\vec{\alpha}}^{1}+\hat{E}_{\vec{\alpha}}^{2} .
\end{aligned}
$$

The super indexes indicate each of the two subsystems on which the operators act. The operators transform the states of the combined system, defined in Eq. (10), in the following way:

$$
\begin{aligned}
& \hat{A}_{i}\left|\vec{\mu}_{1} \vec{M}_{1} ; \vec{\mu}_{2} \vec{M}_{2}\right\rangle=\left(\mu_{i 1}+\mu_{i 2}\right)\left|\vec{\mu}_{1} \vec{M}_{1} ; \vec{\mu}_{2} \vec{M}_{2}\right\rangle, \\
& \hat{E}_{\vec{\alpha}}\left|\vec{\mu}_{1} \vec{M}_{1} ; \vec{\mu}_{2} \vec{M}_{2}\right\rangle=U_{\vec{\alpha}, \vec{\mu}_{1} \vec{M}_{1}}\left|\left(\vec{\mu}_{1}+\vec{\alpha}\right) \vec{M}_{1} ; \vec{\mu}_{2} \vec{M}_{2}\right\rangle \\
& +U_{\vec{\alpha}, \vec{\mu}_{2} \vec{M}_{2}}\left|\vec{\mu}_{1} \vec{M}_{1} ;\left(\vec{\mu}_{2}+\vec{\alpha}\right) \vec{M}_{2}\right\rangle \text {. }
\end{aligned}
$$

We aim to find a basis of states of the combined system whose components are transformed by the operators in Eqs. (19) and (20) in the same way as in Eqs. (12) and (13). The components of this new basis, characterized by the weight vectors $\vec{\mu}$, are linear combinations of all the states of the combined system that satisfy the condition $\vec{\mu}=\vec{\mu}_{1}+\vec{\mu}_{2}$ :

$$
|\vec{\mu} \vec{M}\rangle=\sum_{\vec{\mu}_{1}} C\left(\vec{M}, \vec{\mu}, \vec{\mu}_{1}\right)\left|\vec{\mu}_{1} \vec{M}_{1} ;\left(\vec{\mu}-\vec{\mu}_{1}\right) \vec{M}_{2}\right\rangle .
$$

The coefficients $C\left(\vec{M}, \vec{\mu}, \vec{\mu}_{1}\right)$ are the so-called ClebschGordan coefficients and the summation runs along all the values of $\vec{\mu}_{1}$ for which there exist a $\vec{\mu}_{2}$ that satisfies $\vec{\mu}=$ $\vec{\mu}_{1}+\vec{\mu}_{2}$.

The vectors $\left|\vec{\mu}_{1} \vec{M}_{1} ;\left(\vec{\mu}-\vec{\mu}_{1}\right) \vec{M}_{2}\right\rangle$ form an orthonormal basis, which imposes the following condition:

$$
\begin{aligned}
& \left\langle\left(\vec{\mu}^{\prime}-\vec{\mu}_{1}^{\prime}\right) \vec{M}_{2}^{\prime} ; \vec{\mu}_{1}^{\prime} \vec{M}_{1}^{\prime} \mid \vec{\mu}_{1} \vec{M}_{1} ;\left(\vec{\mu}-\vec{\mu}_{1}\right) \vec{M}_{2}\right\rangle \\
& \quad=\delta_{\vec{M}_{1} \vec{M}_{1}^{\prime}} \delta_{\vec{M}_{2} \vec{M}_{2}^{\prime}} \delta_{\vec{\mu} \vec{\mu}^{\prime}} \delta_{\vec{\mu}_{1} \vec{\mu}_{1}^{\prime}} .
\end{aligned}
$$

From this property, we obtain a definition of the ClebschGordan coefficients:

$$
C\left(\vec{M}, \vec{\mu}, \vec{\mu}_{1}\right)=\left\langle\left(\vec{\mu}-\vec{\mu}_{1}\right) \vec{M}_{2} ; \vec{\mu}_{1} \vec{M}_{1} \mid \vec{\mu} \vec{M}\right\rangle .
$$

The vectors $|\vec{\mu} \vec{M}\rangle$ also form an orthonormal basis:

$$
\left\langle\vec{\mu}^{\prime} \vec{M}^{\prime} \mid \vec{\mu} \vec{M}\right\rangle=\delta_{\vec{M} \vec{M}^{\prime}} \delta_{\vec{\mu} \vec{\mu}^{\prime}}
$$

This condition, combined with the definition of the ClebschGordan coefficients in Eq. (25), leads to the following 
expression for the components of the old basis in terms of the new one:

$$
\left|\vec{\mu}_{1} \vec{M}_{1} ;\left(\vec{\mu}-\vec{\mu}_{1}\right) \vec{M}_{2}\right\rangle=\sum_{\vec{M}} C\left(\vec{M}, \vec{\mu}, \vec{\mu}_{1}\right)|\vec{\mu} \vec{M}\rangle .
$$

From Eqs. (26), (25), and (27) it is possible to obtain the following relationship between the Clebsch-Gordan coefficients:

$$
\sum_{\vec{M}} C\left(\vec{M}, \vec{\mu}, \vec{\mu}_{1}\right) C\left(\vec{M}, \vec{\mu}, \vec{\mu}_{1}^{\prime}\right)=\delta_{\vec{\mu}_{1} \vec{\mu}_{1}^{\prime}}
$$

Operating on the vector $|\vec{\mu} \vec{M}\rangle$ with a ladder operator, and using Eq. (23) to expand the obtained vector $|(\vec{\mu}+\vec{\alpha}) \vec{M}\rangle$, we obtain the expression

$$
\hat{E}_{\vec{\alpha}}|\vec{\mu} \vec{M}\rangle=U_{\vec{\alpha}, \vec{\mu} \vec{M}} \sum_{\vec{\mu}_{1}} C\left(\vec{M}, \vec{\mu}+\vec{\alpha}, \vec{\mu}_{1}\right)\left|\vec{\mu}_{1} \vec{M}_{1} ;\left(\vec{\mu}+\vec{\alpha}-\vec{\mu}_{1}\right)\right\rangle .
$$

On the other hand, first expanding the vector $|\vec{\mu} \vec{M}\rangle$ and then applying the operator,

$$
\begin{aligned}
\hat{E}_{\vec{\alpha}}|\vec{\mu} \vec{M}\rangle= & \sum_{\vec{\mu}_{1}} C\left(\vec{M}, \vec{\mu}, \vec{\mu}_{1}\right)\left(U_{\vec{\alpha}, \vec{\mu}_{1} \vec{M}_{1}}\left|\left(\vec{\mu}_{1}+\vec{\alpha}\right) \vec{M}_{1} ; \vec{\mu}_{2} \vec{M}_{2}\right\rangle\right. \\
& \left.+U_{\vec{\alpha}, \vec{\mu}_{2} \vec{M}_{2}}\left|\vec{\mu}_{1} \vec{M}_{1} ;\left(\vec{\mu}_{2}+\vec{\alpha}\right) \vec{M}_{2}\right\rangle\right) .
\end{aligned}
$$

Comparing Eqs. (29) and (30) and using the orthogonality condition between the vectors, it is possible to derive the following relation:

$$
\begin{aligned}
U_{\vec{\alpha}, \vec{\mu} \vec{M}} C\left(\vec{M}, \vec{\mu}+\vec{\alpha}, \vec{\mu}_{1}\right)= & U_{\vec{\alpha}, \vec{\mu}_{2} \vec{M}_{2}} C\left(\vec{M}, \vec{\mu}, \vec{\mu}_{1}\right) \\
& +U_{\vec{\alpha},\left(\vec{\mu}_{1}-\vec{\alpha}\right) \vec{M}_{1}} C\left(\vec{M}, \vec{\mu}, \vec{\mu}_{1}-\vec{\alpha}\right)
\end{aligned}
$$

\section{Symmetry of energy conserving interactions}

Fermi's golden rule imposes the condition of energy conservation for a nonzero transition rate between states. The operator that describes the energy interaction between two subsystems $\hat{V}_{12}$ does not commute with the Hamiltonian $h_{12}=h_{1}+h_{2}$; however, it is possible to define an operator $\hat{V}_{12}^{\prime}$ that satisfies the following condition:

$$
\left\langle l^{\prime} ; r^{\prime}\left|\hat{V}_{12}^{\prime}\right| r ; l\right\rangle=\left\langle l^{\prime} ; r^{\prime}\left|\hat{V}_{12}\right| r ; l\right\rangle \delta\left(\varepsilon_{r^{\prime} l^{\prime}}-\varepsilon_{r l}\right) .
$$

This new operator commutes with $h_{12}$ and could be expressed as a linear combination of the generators of its symmetry group:

$$
\hat{V}_{12}^{\prime}=\sum_{i} \kappa_{i} \hat{A}_{i}+\sum_{\vec{\alpha}} \kappa_{\vec{\alpha}} \hat{E}_{\vec{\alpha}}
$$

From the previous definition and the condition set in Eq. (32), we obtain

$$
\left\langle\vec{\mu}^{\prime} \vec{M}^{\prime}\left|\hat{V}_{12}\right| \vec{\mu} \vec{M}\right\rangle=\kappa_{\vec{\alpha}} U_{\vec{\alpha}, \vec{\mu} \vec{M}} \delta_{\vec{M} \vec{M}^{\prime}} \delta_{(\vec{\mu}+\vec{\alpha}) \vec{\mu}^{\prime}}
$$

This means that the only allowed transitions are those satisfying the condition $\vec{\mu}^{\prime}=\vec{\mu}+\vec{\alpha}$, for any of the roots of the system. Using Eqs. (27) and (34), we obtain the following equality:

$$
\begin{aligned}
& \left\langle\vec{\mu}_{1}^{\prime} \vec{M}_{1}^{\prime} ;\left(\vec{\mu}^{\prime}-\vec{\mu}_{1}^{\prime}\right) \vec{M}_{2}^{\prime}\left|\hat{V}_{12}\right| \vec{\mu}_{1} \vec{M}_{1} ;\left(\vec{\mu}-\vec{\mu}_{1}\right) \vec{M}_{2}\right\rangle \\
& \quad=\kappa_{\vec{\alpha}} \sum_{\vec{M}} U_{\vec{\alpha}, \vec{\mu} \vec{M}} C\left(\vec{M}, \vec{\mu}+\vec{\alpha}, \vec{\mu}_{1}^{\prime}\right) C\left(\vec{M}, \vec{\mu}, \vec{\mu}_{1}\right) .
\end{aligned}
$$

Equation (31) can be used to transform the summation in Eq. (35) as follows:

$$
\begin{aligned}
& \sum_{\vec{M}} U_{\vec{\alpha}, \vec{\mu} \vec{M}} C\left(\vec{M}, \vec{\mu}+\vec{\alpha}, \vec{\mu}_{1}^{\prime}\right) C\left(\vec{M}, \vec{\mu}, \vec{\mu}_{1}\right) \\
& =U_{\vec{\alpha}, \vec{\mu}_{2} \vec{M}_{2}} \sum_{\vec{M}} C\left(\vec{M}, \vec{\mu}, \vec{\mu}_{1}^{\prime}\right) C\left(\vec{M}, \vec{\mu}, \vec{\mu}_{1}\right) \\
& +U_{\vec{\alpha},\left(\vec{\mu}_{1}^{\prime}-\vec{\alpha}\right) \vec{M}_{1}} \sum_{\vec{M}} C\left(\vec{M}, \vec{\mu}, \vec{\mu}_{1}^{\prime}-\vec{\alpha}\right) C\left(\vec{M}, \vec{\mu}, \vec{\mu}_{1}\right) .
\end{aligned}
$$

Using Eq. (28) we obtain the final expression:

$$
\begin{gathered}
\left\langle\vec{\mu}_{1}^{\prime} \vec{M}_{1}^{\prime} ;\left(\vec{\mu}^{\prime}-\vec{\mu}_{1}^{\prime}\right) \vec{M}_{2}^{\prime}\left|\hat{V}_{12}\right| \vec{\mu}_{1} \vec{M}_{1} ;\left(\vec{\mu}-\vec{\mu}_{1}\right) \vec{M}_{2}\right\rangle \\
=\kappa_{\vec{\alpha}}\left(U_{\vec{\alpha}, \vec{\mu}_{2} \vec{M}_{2}} \delta_{\vec{\mu}_{1} \vec{\mu}_{1}^{\prime}}+U_{\vec{\alpha},\left(\vec{\mu}_{1}^{\prime}-\vec{\alpha}\right) \vec{M}_{1}} \delta_{\vec{\mu}_{1}\left(\vec{\mu}_{1}^{\prime}-\vec{\alpha}\right)}\right) .
\end{gathered}
$$

This means that only transitions satisfying (for any root of the system) $\vec{\mu}_{1}^{\prime}=\vec{\mu}_{1}+\vec{\alpha}$ or $\vec{\mu}_{1}^{\prime}=\vec{\mu}_{1}$ are allowed. The second condition is equivalent to $\vec{\mu}_{2}^{\prime}=\vec{\mu}_{2}+\vec{\alpha}$, as the condition $\vec{\mu}^{\prime}=$ $\vec{\mu}+\vec{\alpha}$ must also be satisfied. Therefore, for nondistinguishable subsystems, each elementary transition involves the change of a single subsystem by one of the roots of the root system that characterized the symmetry group of its Hamiltonian. The transition is driven by a second subsystem that remains unchanged.

To keep the condition of microscopic irreversibility,

$$
\begin{aligned}
& \left\langle\vec{\mu}_{1}^{\prime} \vec{M}_{1}^{\prime} ;\left(\vec{\mu}^{\prime}-\vec{\mu}_{1}^{\prime}\right) \vec{M}_{2}^{\prime}\left|\hat{V}_{12}\right| \vec{\mu}_{1} \vec{M}_{1} ;\left(\vec{\mu}-\vec{\mu}_{1}\right) \vec{M}_{2}\right\rangle \\
& \quad=\left\langle\vec{\mu}_{1} \vec{M}_{1} ;\left(\vec{\mu}-\vec{\mu}_{1}\right) \vec{M}_{2}\left|\hat{V}_{12}\right| \vec{\mu}_{1}^{\prime} \vec{M}_{1}^{\prime} ;\left(\vec{\mu}^{\prime}-\vec{\mu}_{1}^{\prime}\right) \vec{M}_{2}^{\prime}\right\rangle .
\end{aligned}
$$

The following condition should be satisfied:

$$
\kappa_{\vec{\alpha}} U_{\vec{\alpha}, \vec{\mu}}=\kappa_{-\vec{\alpha}} U_{-\vec{\alpha},(\vec{\mu}+\vec{\alpha})} .
$$

Taking Eq. (18) into account, this is equivalent to

$$
\kappa_{\vec{\alpha}}=\kappa_{-\vec{\alpha}} .
$$

This comes from the fact that the operators $\hat{E}_{\vec{\alpha}}$ are not Hermitian, but $\hat{E}_{\vec{\alpha}}$ and $\hat{E}_{-\vec{\alpha}}$ are Hermitian adjoint and therefore their sum is Hermitian. So, in order for the operator $\hat{V}_{12}^{\prime}$ to be Hermitian, the operators $\hat{E}_{\vec{\alpha}}$ and $\hat{E}_{-\vec{\alpha}}$ should appear multiplied by the same coefficient in Eq. (33).

\section{E. Relaxation dynamics of a nonequilibrium system}

Using the previously mentioned results, the transition rate from a state $\vec{\mu} \vec{M}$ to a state $(\vec{\mu}+\vec{\alpha}) \vec{M}$ can be obtained from Eqs. (8) and (37):

$$
T_{\vec{\mu} \vec{M} \rightarrow(\vec{\mu}+\vec{\alpha}) \vec{M}}=\Theta^{2} \Lambda N_{\vec{\mu} \vec{M}}\left(N_{(\vec{\mu}+\vec{\alpha}) \vec{M}}+1\right) \kappa_{\vec{\alpha}}^{2} U_{\vec{\alpha}, \vec{\mu} \vec{M}}^{2} .
$$

The coefficient $\Theta$ is the factor that appears in Eq. (8):

$$
\Theta=\frac{\prod_{\vec{\mu} \vec{M}} N_{\vec{\mu} \vec{M}}}{s !} .
$$

The product in Eq. (42) goes through all the states of the system. The coefficient $\Lambda$ accounts for the interactions with 
all the other subsystems and takes the form

$$
\Lambda=\frac{2 \pi}{\hbar} \sum_{\vec{\mu} \vec{M}} N_{\vec{\mu} \vec{M}}\left(N_{\vec{\mu} \vec{M}}+1\right) .
$$

In Appendix E, we show that if we define the thermodynamic fluxes of the system as the variation rates of the observables associated with quantum operators that belong to the Cartan subalgebra of the Hamiltonian's symmetry group, the following relation between thermodynamic forces and occupation numbers can be established:

$$
\ln \frac{N_{\vec{\mu} \vec{M}}\left(1+N_{(\vec{\mu}+\vec{\alpha}) \vec{M}}\right)}{N_{(\vec{\mu}+\vec{\alpha}) \vec{M}}\left(1+N_{\vec{\mu} \vec{M}}\right)}=\frac{1}{k_{B}} \sum_{i} X_{i} \alpha_{i} .
$$

The notation in Eq. (44) has been changed from Appendix E, in order to fit our description in terms of weights and roots. The coefficients $X_{i}$ are each of the thermodynamic forces of the system.

Equation (44) allows us to obtain the net transition rate from a state $\vec{\mu} \vec{M}$ to a state $(\vec{\mu}+\vec{\alpha}) \vec{M}$ after subtracting the transitions in the opposite sense:

$$
\begin{aligned}
T_{\vec{\mu} \vec{M}} \rightarrow(\vec{\mu}+\vec{\alpha}) \vec{M} & -T_{(\vec{\mu}+\vec{\alpha}) \vec{M} \rightarrow \vec{\mu} \vec{M}} \\
& =\Theta^{2} \Lambda\left(1-e^{-\frac{1}{k_{B}} \sum_{i} X_{i} \alpha_{i}}\right) N_{\vec{\mu} \vec{M}}\left(N_{(\vec{\mu}+\vec{\alpha}) \vec{M}}+1\right) \kappa_{\vec{\alpha}}^{2} U_{\vec{\alpha}, \vec{\mu} \vec{M}}^{2} .
\end{aligned}
$$

Now we are going to compute the rate of change of the occupation number $N_{\vec{\mu} \vec{M}}$ :

$$
\begin{aligned}
\frac{d N_{\vec{\mu} \vec{M}}}{d t}= & \sum_{k}\left(T_{\left(\vec{\mu}-\vec{\alpha}_{k}\right) \vec{M} \rightarrow \vec{\mu} \vec{M}}-T_{\vec{\mu} \vec{M} \rightarrow\left(\vec{\mu}-\vec{\alpha}_{k}\right) \vec{M}}\right. \\
& \left.-T_{\vec{\mu} \vec{M} \rightarrow\left(\vec{\mu}+\vec{\alpha}_{k}\right) \vec{M}}+T_{\left(\vec{\mu}+\vec{\alpha}_{k}\right) \vec{M} \rightarrow \vec{\mu} \vec{M}}\right) .
\end{aligned}
$$

The summation is extended to all the members of the root system (each of them labeled by a different value of the subindex $k$ ), excluding the root $-\vec{\alpha}_{k}$ if $\vec{\alpha}_{k}$ is already included.

As it is shown in Appendix $E$, the occupation number $N_{\vec{\mu} \vec{M}}$ can be expressed using the following equation (using the current notation in terms of group representations), using the subindex $r$ instead of $\vec{\mu} \vec{M}$ to simplify the expression:

$$
N_{r}=\frac{1}{e^{-\alpha+\beta \varepsilon_{\vec{M}}-\frac{1}{k_{B}} \sum_{i} X_{i} \mu_{i}}-1} .
$$

Assuming the values of $\alpha_{i}$ are small, we can make an approximation that will simplify our analysis remarkably:

$$
\begin{aligned}
N_{(\vec{\mu}-\vec{\alpha}) \vec{M}}\left(N_{\vec{\mu} \vec{M}}+1\right) & \approx N_{\vec{\mu} \vec{M}}\left(N_{(\vec{\mu}+\vec{\alpha}) \vec{M}}+1\right) \\
& \approx N_{\vec{\mu} \vec{M}}\left(N_{\vec{\mu} \vec{M}}+1\right) .
\end{aligned}
$$

Equations (17), (45), and (48) allow rewriting Eq. (46) in a very compact way:

$$
\begin{aligned}
\frac{d N_{r}}{d t}= & \sum_{k} \Theta^{2} \Lambda\left(1-e^{-\frac{1}{k_{B}} \sum_{i} X_{i} \alpha_{k i}}\right) N_{r} \\
& \times\left(N_{r}+1\right) \kappa_{\vec{\alpha}_{k}}^{2}\left(\sum_{i} \mu_{r i} \alpha_{k i}\right) .
\end{aligned}
$$

If we assume that the changes of the occupation numbers are driven by changes in the thermodynamic forces of the system, we can obtain the previous derivative directly from Eq. (47):

$$
\frac{d N_{r}}{d t}=\frac{1}{k_{B}} N_{r}\left(N_{r}+1\right) \sum_{i} \mu_{r i} \frac{d X_{i}}{d t} .
$$

Comparing the two previous equations, we obtain differential equations describing the relaxation dynamics of the system:

$$
\frac{d X_{i}}{d t}=\Theta^{2} \Lambda k_{B} \sum_{k}\left(1-e^{-\frac{1}{k_{B}} \sum_{j} X_{j} \alpha_{k j}}\right) \kappa_{\vec{\alpha}_{k}}{ }^{2} \alpha_{k i} .
$$

Equation (51) is not based in any assumption about the proximity of the system to thermodynamic equilibrium and provides a general solution to the problem of the relaxation dynamics of thermodynamic systems.

Linearizing the term between brackets (which for many systems is feasible even far from equilibrium, given the small values of $\alpha_{i}$ ), we would obtain the following system of linear differential equations:

$$
\frac{d X_{i}}{d t}=-\Theta^{2} \Lambda \sum_{j}\left(\sum_{k} \alpha_{k j} \kappa_{\vec{\alpha}_{k}}^{2} \alpha_{k i}\right) X_{j} .
$$

This means that the trajectory toward equilibrium of the system, in the space of thermodynamic forces, follows the negative gradient of the function:

$$
F=\frac{1}{2} \sum_{i j}\left(\sum_{k} \alpha_{k j} \kappa_{\vec{\alpha}_{k}}{ }^{2} \alpha_{k i}\right) X_{i} X_{j}
$$

The parameters $\Theta$ and $\Lambda$ are dependent on the thermodynamic forces of the system and reach a minimum at equilibrium (their derivatives with respect to the thermodynamic forces become zero at equilibrium).

The thermodynamic fluxes (total rates of change of nonconserved observables, as discussed in Appendix E) can be expressed as follows (for any observable associated to the operators in the Cartan algebra):

$$
J_{f}=\frac{d\left\langle\mu_{f}\right\rangle}{d t}
$$

in which

$$
\left\langle\mu_{f}\right\rangle=\sum_{r} \mu_{r f} N_{r}
$$

Equation (54) can be expanded as

$$
J_{f}=\sum_{i}\left(\frac{\partial\left\langle\mu_{f}\right\rangle}{\partial X_{i}}\right) \frac{d X_{i}}{d t} .
$$

We can define the matrix $A$ as follows:

$$
A=\sum_{k} \kappa_{\vec{\alpha}_{k}}^{2}\left(\vec{\alpha}_{k} \otimes \vec{\alpha}_{k}\right) .
$$

Combining the previous definition with Eqs. (52) and (56), the thermodynamic fluxes can be rewritten as

$$
J_{f}=-\Theta^{2} \Lambda \sum_{i}\left(\frac{\partial\left\langle\mu_{f}\right\rangle}{\partial X_{i}}\right) \sum_{j} A_{i j} X_{j} .
$$


Taking the partial derivative of the flux $J_{f}$ with respect to the force $X_{l}$, we obtain

$$
\begin{aligned}
\left(\frac{\partial J_{f}}{\partial X_{l}}\right)= & -\sum_{i} \frac{\partial}{\partial X_{l}}\left[\Theta^{2} \Lambda\left(\frac{\partial\left\langle\mu_{f}\right\rangle}{\partial X_{i}}\right)\right] \sum_{j} A_{i j} X_{j} \\
& -\sum_{i} \Theta^{2} \Lambda\left(\frac{\partial\left\langle\mu_{f}\right\rangle}{\partial X_{i}}\right) A_{i l} .
\end{aligned}
$$

If we compute the derivative in equilibrium, with all the thermodynamic forces equal to zero, the first term in Eq. (59) disappears. On the other hand, deriving Eq. (47), we can obtain the following expression:

$$
\left(\frac{\partial N_{r}}{\partial X_{i}}\right)=\frac{1}{k_{B}} N_{r}\left(N_{r}+1\right) \mu_{r i} .
$$

Combining Eqs. (55) and (60), the following equation is obtained:

$$
\left(\frac{\partial\left\langle\mu_{f}\right\rangle}{\partial X_{l}}\right)=\frac{1}{k_{B}} \sum_{r} N_{r}\left(N_{r}+1\right) \mu_{r l} \mu_{r f} .
$$

If the occupation numbers follow the equilibrium distribution, they depend only on the energy level (and each energy level corresponds to a representation of the symmetry group). Using again the subindex $\vec{M}$ to label energy levels and using the subindex $r$ to label different states within the same energy level, we could rewrite Eq. (61) for the particular case of proximity to equilibrium:

$$
\left(\frac{\partial\left\langle\mu_{f}\right\rangle}{\partial X_{l}}\right)_{\vec{X}=\overrightarrow{0}}=\frac{1}{k_{B}} \sum_{\vec{M}} N_{\vec{M}}\left(N_{\vec{M}}+1\right) \sum_{r \in \vec{M}} \mu_{r l} \mu_{r f} .
$$

Now we remark that for every weight vector $\vec{\mu}_{r}$ in a representation $\vec{M}$, the weight vector $-\vec{\mu}_{r}$ also belongs to the same representation. This fact makes that the summation in Eq. (62) becomes zero except for $l=f$. Therefore, Eq. (59) in equilibrium, takes the form

$$
\left(\frac{\partial J_{f}}{\partial X_{l}}\right)_{\vec{X}=\overrightarrow{0}}=-\Theta^{2} \Lambda\left(\frac{\partial\left\langle\mu_{f}\right\rangle}{\partial X_{f}}\right)_{\vec{X}=\overrightarrow{0}} A_{f l} .
$$

Finally, considering that the set of observables $\left\{\mu_{i}\right\}$ are the eigenvalues of the operators forming the Cartan subalgebra defined in Eq. (10) and that the only properties required for these operators are that they commute with the Hamiltonian of the subsystems and between each other, each observable could be multiplied by an arbitrary constant without loss of generality. The observables can be chosen so that the following condition is satisfied:

$$
\left(\frac{\partial\left\langle\mu_{f}\right\rangle}{\partial X_{f}}\right)_{\vec{X}=\overrightarrow{0}}=\frac{1}{k_{B}} \forall f
$$

Boltzmann's constant has been introduced to keep consistent the units. With this choice of observables, Eq. (63) gives us an expression for Onsager's coefficients, which are indeed symmetric, given the fact that the matrix $A$ is symmetric:

$$
L_{f l}=-\frac{\Theta^{2} \Lambda}{k_{B}} A_{f l} .
$$

\section{DISCUSSION}

The strong constraints imposed by the symmetries of the Hamiltonian $h_{i}$ on the allowed transitions and their relative rates allowed us to obtain Eq. (52), whose coefficients can be calculated from the symmetries of the Hamiltonian $h_{i}$ and the form of the interaction potential between subsystems. Equation (52) fully describes the relaxation toward equilibrium of a thermodynamic system composed by identical subsystems (and these subsystems can be chosen to be as complex as we want without changing the general conclusions). The thermodynamic fluxes (rates of change of macroscopic observables) can be computed using Eq. (55). Equation (53) shows that there is indeed a potential whose negative gradient determines the trajectory of the system and whose minimal value corresponds to a state of maximal entropy. However, the particular shape of this potential depends on the system being studied and its symmetries.

Nonequilibrium steady states, resulting from externally imposed constraints on the thermodynamic forces, could be obtained by minimizing the potential $F$ subject to these constraints.

Equation (65) shows a relation between Onsager's coefficients and the structure of the symmetry group of the system (which determines the matrix A); this allows both predicting the coefficients $a b$ initio based on the interaction potential between subsystems or using experimentally measured coefficients (in the proximity of equilibrium), to obtain matrix A and use it to model the relaxation of the system also arbitrarily far from equilibrium, by using Eqs. (51) and (52).

\section{APPENDIX A: TRANSITIONS BETWEEN STATES FOR DISTINGUISHABLE SUBSYSTEMS}

In this section, we are going to assume that the subsystems are distinguishable between each other, and therefore the wave function $|\sigma\rangle$ is different from the function $|p(\sigma)\rangle$, where $p(\sigma)$ is a permutation of the indexes in the s-tuple $\sigma$. As it will be shown later, the consideration of distinguishable subsystems will lead us to the derivation of Boltzmann's distribution, while the assumption of nondistinguishable systems will lead to the Bose-Einsten distribution. When considering nonequilibrium thermodynamics, we will see that the two approaches lead to different dynamic properties of the system.

The functions $|\sigma\rangle$ are eigenvectors of the operator $\hat{H}_{0}=$ $\sum_{i=1}^{s} h_{i}$. In the absence of interactions between the subsystems this operator would be the Hamiltonian of the whole system, and if this system is initially observed in the state $\left|\sigma_{0}\right\rangle$, then it will always be observed in the same state and there would not be entropy change. If there are interactions between the subsystems, then the Hamiltonian of the whole system will include an extra term accounting for these interactions:

$$
\hat{H}=\sum_{i} h_{i}+\sum_{i, j} \frac{1}{2} \hat{V}_{i j}=\hat{H}_{0}+\hat{V} .
$$

The functions $|\sigma\rangle$ are not eigenvectors of the complete Hamiltonian of the system, and it is therefore possible to observe the system in a state $\left|\sigma_{0}\right\rangle$ and, after a certain time $t$, observe it in a different state $\left|\sigma_{f}\right\rangle$ with possibly different 
entropy. If we assume the interactions between subsystems to be weak in comparison to the interactions within each subsystem, we can obtain an expression for the probability of this event in the same way as Fermi's golden rule is derived.

According to Fermi's golden rule [11,12], the frequency of observed transitions per unit of time between two states is described by the expression

$$
W_{\sigma_{0} \rightarrow \sigma_{f}}=\frac{2 \pi}{\hbar}\left\langle\sigma_{f}|\hat{V}| \sigma_{0}\right\rangle^{2} \delta\left(E_{\sigma_{f}}-E_{\sigma_{0}}\right) .
$$

The parameters $E_{\sigma_{0}}$ and $E_{\sigma_{f}}$ are the corresponding eigenvalues of the operator $\hat{H}_{0}$ and $\delta$ is Dirac's function.

We remind that the functions $\left|\sigma_{0}\right\rangle$ and $\left|\sigma_{f}\right\rangle$ take the form of Eq. (4). We will use the following notation:

$$
\begin{gathered}
\left|\sigma_{0}\right\rangle=\left|1_{k_{1}^{0}}\right\rangle\left|2_{k_{2}^{0}}\right\rangle \ldots\left|s_{k_{s}^{0}}\right\rangle, \\
\left|\sigma_{f}\right\rangle=\left|1_{k_{1}} f\right\rangle\left|2_{k_{2}}{ }^{f}\right\rangle \ldots\left|s_{k_{s}}{ }^{f}\right\rangle .
\end{gathered}
$$

The coefficients $\left\langle\sigma_{f}|\hat{V}| \sigma_{0}\right\rangle$ can be computed as follows:

$$
\left\langle\sigma_{f}|\hat{V}| \sigma_{0}\right\rangle=\frac{1}{2} \sum_{i, j}\left(\left\langle i_{k_{i} f}\left|\left\langle j_{k_{j}^{f}}\left|\hat{V}_{i j}\right| j_{k_{j}^{0}}\right\rangle\right| i_{k_{i}^{0}}\right\rangle \prod_{u \neq i, j}\left\langle u_{k_{u}^{f}} \mid u_{k_{u}^{0}}\right\rangle\right) .
$$

Note that if $k_{u}^{f} \neq k_{u}^{0}$, then $\left\langle u_{k_{u} f} \mid u_{k_{u}{ }^{0}}\right\rangle=0$.

This means that if three or more subsystems differ between the initial and final state, all the terms in the summation are zero and no transitions between both states are observed. In other words, the observed transitions involve the change of at most two subsystems, and they are driven by the interaction between these two subsystems. We can therefore use the following notation for the transition frequencies:

$$
\begin{aligned}
W_{\sigma_{0} \rightarrow \sigma_{f}} & =W_{\left(k_{i}^{0}, k_{j}^{0}\right) \rightarrow\left(k_{i}^{f}, k_{j}^{f}\right)} \\
& =\frac{2 \pi}{\hbar}\left\langle i_{k_{i}^{f}}\left|\left\langle j_{k_{j}^{f}}\left|\hat{V}_{i j}\right| j_{k_{j}}{ }^{0}\right\rangle\right| i_{k_{i}}\right\rangle^{2} \delta\left(E_{\sigma_{f}}-E_{\sigma_{0}}\right) .
\end{aligned}
$$

The presence of the function $\delta$ of Dirac also imposes the following energy-conservation condition:

$$
\varepsilon_{k_{i}^{0}}+\varepsilon_{k_{j}^{0}}=\varepsilon_{k_{i}^{f}}+\varepsilon_{k_{j}^{f}},
$$

or

$$
\Delta \varepsilon_{i}=-\Delta \varepsilon_{j}
$$

Note that as the subsystems have been chosen to be identical, the form of the operators $\hat{V}_{i j}$ is the same, and only the variables on which they act change. So, we can, in general, write

$$
W_{\left(k_{i}^{0}, k_{j}^{0}\right) \rightarrow\left(k_{i}^{f}, k_{j}^{f}\right)}=W_{\left(r \rightarrow r^{\prime}, l \rightarrow l^{\prime}\right)} .
$$

This just means that the transitions in which a subsystem $I$ in the state $k_{i}{ }^{0}=r$ passes to a state $k_{i}{ }^{f}=r^{\prime}$ and a second subsystem $j$ in the state $k_{j}{ }^{0}=l$ passes to the state $k_{j}{ }^{f}=l^{\prime}$, occur with the same frequency independently of which are the subsystems $I$ and $j$.

Now, if we have a system with $N_{r}$ subsystems in the state $r$ and $N_{l}$ subsystems in the state $l$. The total frequency of $\left(r \rightarrow r^{\prime}, l \rightarrow l^{\prime}\right)$ transitions will be

$$
T_{\left(r \rightarrow r^{\prime}, l \rightarrow l^{\prime}\right)}=N_{r} N_{l} W_{\left(r \rightarrow r^{\prime}, l \rightarrow l^{\prime}\right)} .
$$

\section{APPENDIX B: TRANSITIONS BETWEEN STATES FOR NONDISTINGUISHABLE SUBSYSTEMS}

In this case, the wave function describing the whole system has to be symmetric with respect to the permutation of two subsystems. Even if the system is composed of fermions, we can define the subsystems as pairs of fermions; therefore, we can assume symmetric wave functions without loss of generality. Symmetric normalized wave functions will take the following form:

$$
|S\rangle=\sqrt{\frac{\prod_{k} N_{k} !}{s !}} \sum_{p(\sigma)}|p(\sigma)\rangle .
$$

The transition frequency between two states will be as described before:

$$
W_{S_{0} \rightarrow S_{f}}=\frac{2 \pi}{\hbar}\left\langle S_{f}|\hat{V}| S_{0}\right\rangle^{2} \delta\left(E_{S_{f}}-E_{S_{0}}\right) .
$$

In this case,

$\left\langle S_{f}|\hat{V}| S_{0}\right\rangle=\sqrt{\frac{\prod_{k} N_{k f}}{s !}} \sqrt{\frac{\prod_{k} N_{k 0}}{s !}} \sum_{p\left(\sigma_{f}\right), p\left(\sigma_{0}\right)}\left\langle p\left(\sigma_{f}\right)|\hat{V}| p\left(\sigma_{0}\right)\right\rangle$.

In the same way as in Appendix A [Eq. (A5)], we can see that if more than two subsystems change between the initial and final states, the transition frequency between these states becomes zero. If the change between states involves a subsystem in the state $r$ transiting to state $r^{\prime}$ and a subsystem in state $l$ transiting to state $l^{\prime}$, the summation in Eq. (B3) takes the form

$$
\begin{aligned}
& \sum_{p\left(\sigma_{f}\right), p\left(\sigma_{0}\right)}\left\langle p\left(\sigma_{f}\right)|\hat{V}| p\left(\sigma_{0}\right)\right\rangle \\
& \quad=N_{r} N_{l}\left(\Omega_{\left(r \rightarrow r^{\prime}, l \rightarrow l^{\prime}\right)}+\Omega_{\left(r \rightarrow l^{\prime}, l \rightarrow r^{\prime}\right)}\right),
\end{aligned}
$$

where

$$
\Omega_{\left(r \rightarrow r^{\prime}, l \rightarrow l^{\prime}\right)}=\left\langle i_{r^{\prime}}\left|\left\langle j_{l^{\prime}}\left|\hat{V}_{i j}\right| j_{l}\right\rangle\right| i_{r}\right\rangle .
$$

The value of this expression does not depend on the particular subsystems $I$ and $j$ but only on the states between which the transitions occur. Equation (B5) accounts for all the possible combinations of subsystems in states $r$ and $l$, respectively. As the subsystems are now undistinguishable, there is no difference between a situation in which a subsystem in the state $r$ transits to state $r^{\prime}$ and a subsystem in state $l$ transits to state $l^{\prime}$ or a subsystem in state $r$ transits to state $l^{\prime}$ and a subsystem in state $l$ transits to state $r^{\prime}$. That's why both situations are grouped in Eq. (B4). In order to simplify the expression, we will use the following: notation:

$$
\Omega_{\left(r \rightarrow r^{\prime}, l \rightarrow l^{\prime}\right)}+\Omega_{\left(r \rightarrow l^{\prime}, l \rightarrow r^{\prime}\right)}=\Omega_{r l \rightarrow r^{\prime} l^{\prime}}
$$

The parameter $\Omega_{r l \rightarrow r^{\prime} l^{\prime}}$ can be redefined in a more compact way as follows:

$$
\Omega_{r l \rightarrow r^{\prime} l^{\prime}}=\left\langle l^{\prime} ; r^{\prime}\left|\hat{V}_{i j}\right| r ; l\right\rangle,
$$


where

$$
|r ; l\rangle=\frac{1}{\sqrt{2}}\left(\left|i_{r}\right\rangle\left|j_{l}\right\rangle+\left|i_{l}\right\rangle\left|j_{r}\right\rangle\right) .
$$

To simplify Eq. (21), we have to notice the following relations: $\quad N_{r f}=N_{r 0}-1 ; N_{l f}=N_{l 0}-1 ; N_{r^{\prime} f}=N_{r^{\prime} 0}+1$; $N_{l^{\prime} f}=N_{l^{\prime} 0}+1$. For $k \neq r, l, r^{\prime}, l^{\prime} N_{k f}=N_{k 0}$. This allows us to rewrite Eq. (B3) in the following way:

$$
\left\langle S_{f}|\hat{V}| S_{0}\right\rangle=\frac{\prod_{k} N_{k 0}}{s !} \sqrt{N_{r 0} N_{l 0}\left(N_{r^{\prime} 0}+1\right)\left(N_{l^{\prime} 0}+1\right)} \Omega_{r l \rightarrow r^{\prime} l^{\prime}} .
$$

Finally, we can obtain the transition frequency between states as

$$
\begin{aligned}
W_{S_{0} \rightarrow S_{f}}= & \frac{2 \pi}{\hbar}\left(\frac{\prod_{k} N_{k 0}}{s !}\right)^{2} N_{r 0} N_{l 0}\left(N_{r^{\prime} 0}+1\right) \\
& \times\left(N_{l^{\prime} 0}+1\right) \Omega_{r l \rightarrow r^{\prime} l^{\prime}}^{2} \delta\left(E_{S_{f}}-E_{S_{0}}\right) .
\end{aligned}
$$

In this case, as the subsystems are indistinguishable, the states are just defined by the numbers of subsystems in each state; therefore, this value is identical to the frequency of $l r \rightarrow$ $l^{\prime} r^{\prime}$ transitions. Therefore, for nondistinguishable subsystems, the equivalent of Eq. (A10) is just

$$
\begin{aligned}
T_{r l \rightarrow r^{\prime} l^{\prime}}= & \frac{2 \pi}{\hbar}\left(\frac{\prod_{k} N_{k 0}}{s !}\right)^{2} N_{r 0} N_{l 0}\left(N_{r^{\prime} 0}+1\right) \\
& \times\left(N_{l^{\prime} 0}+1\right) \Omega_{r l \rightarrow r^{\prime} l^{\prime}}^{2} \delta\left(E_{S_{f}}-E_{S_{0}}\right) .
\end{aligned}
$$

From now on we will drop the subzero indexes and assume that we are always referring to the occupation numbers of the current state of the system. In a more compact way we can rewrite the previous equation using the following notation:

$$
\begin{gathered}
\Gamma_{r l \rightarrow r^{\prime} l^{\prime}}=\Gamma_{r^{\prime} l^{\prime} \rightarrow r l}=\frac{2 \pi}{\hbar} \Omega_{r l \rightarrow r^{\prime} l^{\prime}}^{2} \delta\left(E_{S_{f}}-E_{S_{0}}\right), \\
T_{r l \rightarrow r^{\prime} l^{\prime}}=\left(\frac{\prod_{k} N_{k}}{s !}\right)^{2} \Gamma_{r l \rightarrow r^{\prime} l^{\prime}} N_{r} N_{l}\left(N_{r^{\prime}}+1\right)\left(N_{l^{\prime}}+1\right) .
\end{gathered}
$$

\section{APPENDIX C: EQUILIBRIUM CONDITION FOR DISTINGUISHABLE SUBSYSTEMS AND BOLTZMANN'S DISTRIBUTION}

Now we will analyze in which conditions the frequency of each transition $\left(r \rightarrow r^{\prime}, l \rightarrow l^{\prime}\right)$ is equal to the frequency of its opposite transition $\left(r^{\prime} \rightarrow r, l^{\prime} \rightarrow l\right)$, which implies that the numbers of subsystems in any state do not change (in average) over time. The equilibrium condition can be expressed as follows:

$$
T_{\left(r \rightarrow r^{\prime}, l \rightarrow l^{\prime}\right)}=T_{\left(r^{\prime} \rightarrow r, l^{\prime} \rightarrow l\right)} .
$$

From Eq. (A6), we see that by definition,

$$
W_{\left(r \rightarrow r^{\prime}, l \rightarrow l^{\prime}\right)}=W_{\left(r^{\prime} \rightarrow r, l^{\prime} \rightarrow l\right)} .
$$

Equation (C2) is just an expression for the property of microscopic reversibility.

The equilibrium condition then becomes just equivalent to

$$
N_{r} N_{l}=N_{r^{\prime}} N_{l^{\prime}},
$$

or

$$
\frac{N_{r}}{N_{r^{\prime}}}=\frac{N_{l^{\prime}}}{N_{l}} .
$$

By taking logarithms, we can just rewrite the equilibrium condition as follows:

$$
\Delta \ln N_{r}=-\Delta \ln N_{l},
$$

where

$$
\Delta \ln N_{r}=\ln N_{r^{\prime}}-\ln N_{r} .
$$

If all the possible transitions between states satisfy Eq. (C5), the system is in equilibrium.

As it is stated in Eq. (A7), all the observable transitions between states satisfy also the energy conservation condition:

$$
\Delta \varepsilon_{r}=-\Delta \varepsilon_{l} .
$$

Equations (C5) and (C7) imply that, in equilibrium, the difference between the energy levels of two states is proportional to the difference between the logarithms of the numbers of subsystems in these states. This implies that there is a linear relation between the logarithm of the number of subsystems in a particular state and the energy of this state:

$$
\ln N_{r}=\alpha-\beta \varepsilon_{r} \forall r,
$$

or

$$
N_{r}=\exp (\alpha) \exp \left(-\beta \varepsilon_{r}\right)
$$

The values of the parameters $\alpha$ and $\beta$ are fixed by specifying the total number of subsystems and the total energy of the system (the sum of the observed eigenvalues of the Hamiltonians of each subsystem). This is identical to Boltzmann's distribution:

$$
\exp (\alpha)=\frac{s}{Z} \beta=\frac{1}{k_{B} T} .
$$

The variable $s$ is the total number of subsystems and $Z$ the partition function of the system.

As it is well known, Boltzmann's equation results from maximizing the entropy of a system by keeping the total energy constrained; therefore, we have just shown that the state of equilibrium (the numbers of subsystems in any state do not change over time) is equivalent to a state of maximal entropy.

\section{APPENDIX D: EQUILIBRIUM CONDITION FOR NONDISTINGUISHABLE SUBSYSTEMS AND BOSE-EINSTEIN DISTRIBUTION}

In the same way as we did for the case of distinguishable subsystems, we set as equilibrium condition the equality between the frequencies of transitions $l r \rightarrow l^{\prime} r^{\prime}$ and $l^{\prime} r^{\prime} \rightarrow l r$. It is very important to remark that this second frequency is not the frequency of transitions back from $S_{f}$ to $S_{0}$ (which would be the same as the previous one) but the frequency of transitions from $S_{0}$ to a state different from $S_{0}$ and $S_{f}$ in which two subsystems in the states $r^{\prime}$ and $l^{\prime}$ have been substituted by two subsystems in states $r$ and $l$. Equation (B13) reveals that the equilibrium condition implies that for all the allowed transitions (satisfying the energy conservation 
condition) the following relation has to be satisfied for every energy conserving transition:

$$
N_{r} N_{l}\left(N_{r^{\prime}}+1\right)\left(N_{l^{\prime}}+1\right)=N_{r^{\prime}} N_{l^{\prime}}\left(N_{r}+1\right)\left(N_{l}+1\right) .
$$

Using the same notation as in Eq. (C6), we can rewrite the previous expression as

$$
\Delta \ln \frac{N_{r}}{N_{r}+1}=-\Delta \ln \frac{N_{l}}{N_{l}+1} .
$$

This leads us to the following relation:

$$
\ln \frac{N_{r}}{N_{r}+1}=\alpha-\beta \varepsilon_{r} \forall r,
$$

or alternatively,

$$
N_{r}=\frac{1}{e^{-\alpha+\beta \varepsilon_{r}}-1} .
$$

This corresponds to Bose-Einstein's distribution and the value of $\alpha$ is adjusted so that the total number of subsystems is equal to the sum of the numbers of subsystems in each state.

As all the degenerate states of a certain energy level have the same occupation numbers, the total number of subsystems in a certain energy level is just

$$
N_{\varepsilon}=\frac{g_{\varepsilon}}{e^{-\alpha+\beta \varepsilon}-1} .
$$

The parameter $g_{\varepsilon}$ is the number of states in the corresponding energy level.

This distribution, as it is well known, corresponds to the maximization at constant energy and particle number (in our case number of subsystems) of the entropy of a system of nondistinguishable particles (subsystems), which is defined as

$$
S=k_{B} \ln \prod_{\varepsilon} \frac{\left(g_{\varepsilon}+N_{\varepsilon}-1\right) !}{\left(g_{\varepsilon}-1\right) ! N_{\varepsilon} !} .
$$

\section{APPENDIX E: STATISTICS OF NONEQUILIBRIUM SYSTEMS}

The content of this Appendix has been largely presented in Ref. [6].

We start by defining thermodynamic fluxes as time derivatives of certain nonconserved observables:

$$
\frac{d \phi_{f}}{d t}=J_{f} .
$$

In this article, we are going to restrict ourselves to observables associated to quantum operators $\hat{\Phi}_{f}$ that commute with the Hamiltonian $\hat{H}_{0}$ :

$$
\left[\hat{H}_{0}, \hat{\Phi}_{f}\right]=0 .
$$

If this was not the case, the time evolution of the observable would be due also in part driven by $\hat{H}_{0}$ and not only by the interactions between subsystems and the transitions between states that we describe in Eqs. (A10) and (B13). This also means that each state corresponding to an eigenvector of $\hat{H}_{0}$ has a well-defined value of the observables $\phi_{f}$ and any transition between eigenvectors is associated to a well-defined increment of the observables $\phi_{f}$.
We are going to proceed in the same way as previously, by describing the cases of distinguishable and nondistinguishable subsystems.

\section{Distinguishable subsystems}

A transition $\left(r \rightarrow r^{\prime}, l \rightarrow l^{\prime}\right)$ is associated to the following entropy change (directly derived from Boltzmann's definition of entropy):

$$
\Delta S_{\left(r \rightarrow r^{\prime}, l \rightarrow l^{\prime}\right)}=k_{B} \ln \frac{N_{r} N_{l}}{\left(N_{r^{\prime}}+1\right)\left(N_{l^{\prime}}+1\right)} .
$$

For large values of the occupation numbers, we can simplify as

$$
\Delta S_{\left(r \rightarrow r^{\prime}, l \rightarrow l^{\prime}\right)} \approx k_{B} \ln \frac{N_{r} N_{l}}{N_{r^{\prime}} N_{l^{\prime}}} .
$$

The entropy increase rate can be obtained as a function of all the transition rates:

$$
\frac{d S}{d t}=k_{B} \sum_{r, r^{\prime}, l, l^{\prime}} T_{\left(r \rightarrow r^{\prime}, l \rightarrow l^{\prime}\right)} \ln \frac{N_{r} N_{l}}{N_{r^{\prime}} N_{l^{\prime}}} .
$$

On the other hand, using a description in terms of thermodynamic fluxes and forces, the entropy production rate will be

$$
\frac{d S}{d t}=\sum_{f} X_{f} J_{f}
$$

If we rewrite the thermodynamic fluxes as functions of the transitions between states, we obtain

$$
J_{f}=\sum_{r, r^{\prime}, l, l^{\prime}} T_{\left(r \rightarrow r^{\prime}, l \rightarrow l^{\prime}\right)} \Delta \phi_{f\left(r \rightarrow r^{\prime}, l \rightarrow l^{\prime}\right)} .
$$

Combining the two previous equations we obtain

$$
\begin{aligned}
\frac{d S}{d t} & =\sum_{f} X_{f} \sum_{r, r^{\prime}, l, l^{\prime}} T_{\left(r \rightarrow r^{\prime}, l \rightarrow l^{\prime}\right)} \Delta \phi_{f\left(r \rightarrow r^{\prime}, l \rightarrow l^{\prime}\right)} \\
& =\sum_{r, r^{\prime}, l, l^{\prime}} T_{\left(r \rightarrow r^{\prime}, l \rightarrow l^{\prime}\right)} \sum_{f} X_{f} \Delta \phi_{f\left(r \rightarrow r^{\prime}, l \rightarrow l^{\prime}\right)} .
\end{aligned}
$$

Comparing Eqs. (E5) and (E8), we can infer that in a thermodynamic system not in equilibrium and characterized by a set of thermodynamic forces, the following equality is satisfied:

$$
\ln \frac{N_{r} N_{l}}{N_{r^{\prime}} N_{l^{\prime}}}=\frac{1}{k_{B}} \sum_{f} X_{f} \Delta \phi_{f\left(r \rightarrow r^{\prime}, l \rightarrow l^{\prime}\right)} .
$$

For the previous equation to be satisfied in all the possible transitions, the occupation numbers of the different states should follow the following distribution:

$$
N_{r}=e^{\alpha-\beta \varepsilon_{r}+\frac{1}{k_{B}} \sum_{f} X_{f} \phi_{f r}} .
$$

\section{Nondistinguishable subsystems}

In this case, the entropy results from the number of ways in which a certain number of nondistinguishable subsystems can be arranged within different boxes containing sets of $g$ states. In Eq. (D6), each of these boxes corresponds to an energy level, that's why the index $\varepsilon$ has been used. Here we define 
the boxes as sets of states with the same energy and the same values of the other observables $\phi_{f}$ used to describe the system. For this reason, we use the more general index $\lambda$ :

$$
S=k_{B} \ln \prod_{\lambda} \frac{\left(g_{\lambda}+N_{\lambda}-1\right) !}{\left(g_{\lambda}-1\right) ! N_{\lambda} !} .
$$

Now let's consider the transition $r l \rightarrow r^{\prime} l^{\prime}$ in which the states $r, r^{\prime}, l$, and $l^{\prime}$ belong to the boxes $\rho, \rho^{\prime}, \lambda$, and $\lambda^{\prime}$, respectively. Using Eq. (E11), we can compute the entropy change associated to this transition:

$$
\begin{aligned}
& \Delta S_{r l \rightarrow r^{\prime} l^{\prime}} \\
& \quad=k_{B} \ln \frac{N_{\rho}\left(g_{\rho^{\prime}}+N_{\rho^{\prime}}\right) N_{\lambda}\left(g_{\lambda^{\prime}}+N_{\lambda^{\prime}}\right)}{\left(N_{\rho^{\prime}}+1\right)\left(g_{\rho}+N_{\rho}-1\right)\left(N_{\lambda^{\prime}}+1\right)\left(g_{\lambda}+N_{\lambda}-1\right)} .
\end{aligned}
$$

For large occupation numbers, we can simplify as

$$
\Delta S_{r l \rightarrow r^{\prime} l^{\prime}} \approx k_{B} \ln \frac{N_{\rho}\left(g_{\rho^{\prime}}+N_{\rho^{\prime}}\right) N_{\lambda}\left(g_{\lambda^{\prime}}+N_{\lambda^{\prime}}\right)}{N_{\rho^{\prime}}\left(g_{\rho}+N_{\rho}\right) N_{\lambda^{\prime}}\left(g_{\lambda}+N_{\lambda}\right)} .
$$

If all the states within each box have the same occupation numbers, we can write

$$
N_{\lambda}=g_{\lambda} N_{l} \text {. }
$$

Doing this substitution for each of the states involved in the transition, we obtain

$$
\Delta S_{r l \rightarrow r^{\prime} l^{\prime}} \approx k_{B} \ln \frac{N_{r}\left(1+N_{r^{\prime}}\right) N_{l}\left(1+N_{l^{\prime}}\right)}{N_{r^{\prime}}\left(1+N_{r}\right) N_{l^{\prime}}\left(1+N_{l}\right)} .
$$

Now we just need to proceed in an identical way as we did for the case of distinguishable subsystems to obtain the identity:

$\ln \frac{N_{r}\left(1+N_{r^{\prime}}\right) N_{l}\left(1+N_{l^{\prime}}\right)}{N_{r^{\prime}}\left(1+N_{r}\right) N_{l^{\prime}}\left(1+N_{l}\right)}=\frac{1}{k_{B}} \sum_{f} X_{f} \Delta \phi_{f\left(r l \rightarrow r^{\prime} l^{\prime}\right)}$.

As in the case of distinguishable subsystems, for the previous equation to be satisfied in all the possible transitions, the distribution of the occupation numbers of each state should follow the equation

$$
N_{r}=\frac{1}{e^{-\alpha+\beta \varepsilon_{r}-\frac{1}{k_{B}} \sum_{f} X_{f} \phi_{f r}}-1} .
$$

[1] G. Lebon, D. Jou and J. Casas-Vázquez. Understanding Nonequilibrium Thermodynamics (Springer, Berlin, 2008).

[2] I. Prigogine, Science 201, 777 (1978).

[3] H. Ziegler, Ing. Arch. 30, 410 (1961).

[4] G. P. Beretta, Phys. Rev. E 90, 042113 (2014).

[5] J. Hurley and C. Garrod, Phys. Rev. Lett. 48, 1575 (1982).

[6] S. Bordel, Physica A 389, 4564 (2010).

[7] S. Bordel, J. Stat. Mech. (2011) P05013.

[8] G. P. Beretta, Found. Phys. 17, 365 (1987).

[9] G. P. Beretta, Rep. Math. Phys. 64, 139 (2009).

[10] G. P. Beretta, Entropy 10, 160 (2008).

[11] M. D. Kostin, J. Chem. Phys. 57, 3589 (1972).
[12] I. Bialyniki-Birula and J. Mycielski, Ann. Phys. 100, 62 (1976).

[13] H. D. Doebner and G. A. Goldin, Phys. Rev. A 54, 3764 (1996).

[14] J. J. Halliwell and J. Perez-Mercader, and W. G. Zurek, Physical Origins of Time Asymmetry (Cambridge University Press, Cambridge, 1996).

[15] L. Maccone, Phys. Rev. Lett. 103, 080401 (2009).

[16] E. Fermi, Nuclear Physics (University of Chicago Press, Chicago, 1950).

[17] D. N. Zubarev and V. P. Kalashnikov, Physica 46, 550 (1970).

[18] R. C. Dewar, J. Phys. A: Math. Gen. 36, 631 (2003).

[19] H. F. Jones, Groups, Representations, and Physics (Taylor \& Francis, London, 1998). 